\title{
Neuro-Thoracic Radiologists "Corner": Incidental Pulmonary Findings on a Neck MRI Leading to the Diagnosis of COVID-19
}

W

e read with great interest the recently published article by Jain et $\mathrm{al}^{1}$ in the July 2020 issue of the American Journal of Neuroradiology. In this article, the authors reported a cohort of 17 patients who presented to the emergency department with either trauma or stroke-like symptoms and underwent neuroimaging evaluation revealing incidental thoracic findings on a CTA of the neck or CT of the cervical/thoracic spine, suspicious for viral pneumonia, triggering testing for Severe Acute Respiratory Syndrome coronavirus 2 (SARS-CoV-2) with an eventual diagnosis of coronavirus disease 2019 (COVID-19). It is emphasized that neuroradiologists should be familiar with the imaging appearance of common thoracic and abdominal diseases that can be detected on neuroimaging studies and they should always carefully scrutinize the image "corners" or "blind-spots" to minimize perceptual diagnostic errors, the significance of which has never been more important. Similarly, as MR imaging outpatient volumes continue to return to normal levels as the country reopens, larger numbers of potentially infected-but-asymptomatic or paucisymptomatic patients will be coming to the radiology clinics each day, providing new opportunities for incidental detection of COVID-19 on routine MR neuroimaging. To date, there is a scarcity of reporting on the MR imaging appearance of the coronavirus disease, ${ }^{2}$ which is not surprising given the overall reduced MR imaging volume and strict infection control measures taken during the lockdown.

We, therefore, report the following case of incidentally detected COVID-19 in a 43-year-old man undergoing MR imaging of the neck. He presented as an outpatient to his otorhinolaryngologist with chronic intermittent throat/neck and ear pain, excessive sputum production, and a history of prior tonsillectomy. Findings of physical examination, throat swab for Streptococcus pyogenes, and nasopharyngolaryngoscopy were negative. MR imaging of the neck was ordered, which showed no aerodigestive tract mass or cervical lymphadenopathy (Fig 1); however, slices through the lung apices showed bilateral, peripheral consolidation with rounded morphology, consistent with

-- Indicates open access to non-subscribers at www.ajnr.org http://dx.doi.org/10.3174/ajnr.A6699

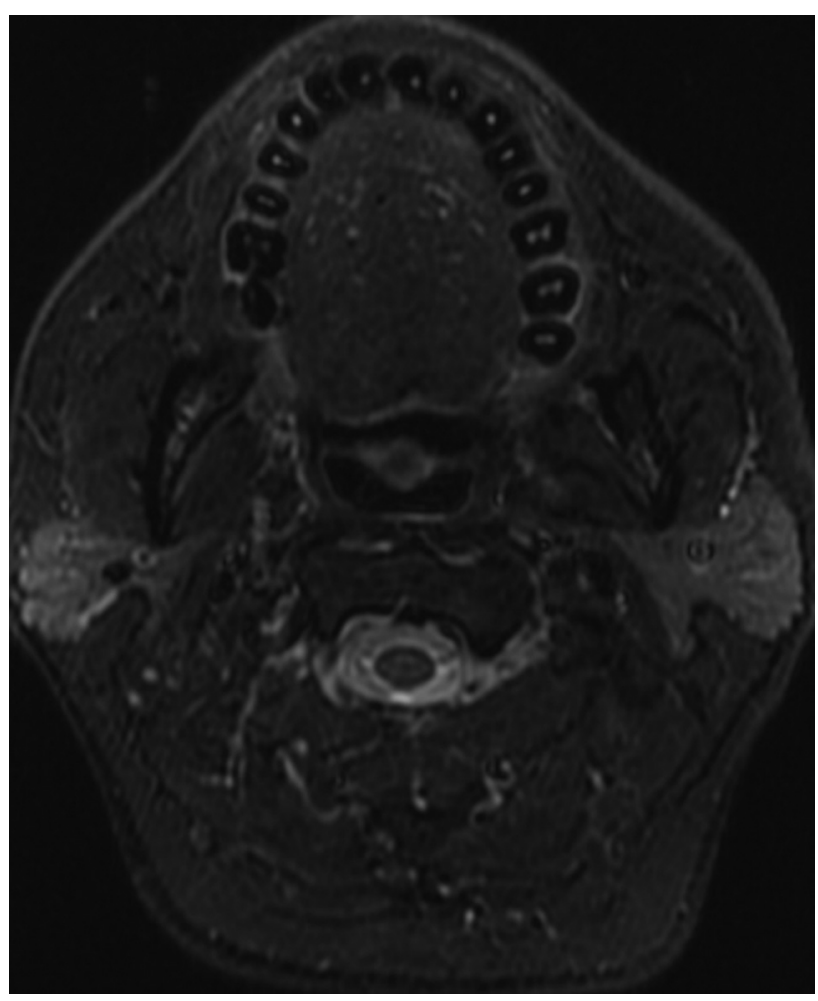

FIG 1. Axial STIR image, at the level of the oropharynx, demonstrating no oropharyngeal mass or abnormality at the site of prior tonsillectomy.

high confidence features of COVID-19 pneumonia (Fig 2). The consolidation was hyperintense on T2/STIR sequences and avidly enhanced on T1-weighted fast multiplanar spoiled gradient-echo sequences. These findings were urgently communicated to the ordering otorhinolaryngologist with a recommendation for SARS-CoV-2 testing. The patient tested positive the next day, and later remembered mild upper respiratory tract infection symptoms a few weeks prior. Retrospectively, the presenting symptoms may have been an atypical manifestation of COVID19.

The cases highlighted by Jain et $\mathrm{al}^{1}$ and our example above demonstrate that neuroradiologists can be the first to detect 

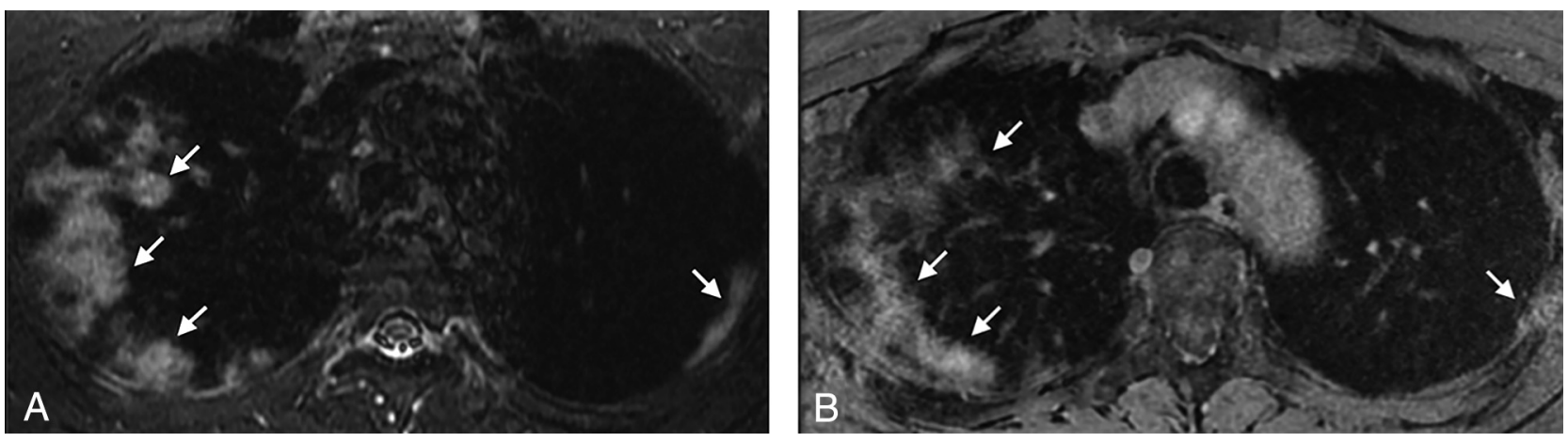

FIG 2. Axial STIR image $(A)$ and axial postcontrast T1-weighted image obtained with a fast multiplanar spoiled gradient-echo sequence (B) through the lung apices demonstrating bilateral, peripheral consolidation with rounded morphology, consistent with high confidence features of COVID-19 pneumonia (arrows).

incidental pulmonary findings of major importance leading to an unsuspected diagnostic discovery. This detection is becoming even more relevant during the current coronavirus pandemic when many patients are presenting with atypical symptoms that prompt neurologic or body imaging studies on which incidental thoracic findings may yield an accurate and expedited diagnosis that could be lifesaving for patients and prevent spread of the disease. The role of neuroradiologists during this pandemic is evolving with detection of a spectrum of CNS abnormalities that were not previously known, ${ }^{3}$ as well as heightened sensitivity for incidental pulmonary findings leading to an expanding repertoire of our specialty as neuro-thoracic radiologists.

Disclosures: Suyash Mohan—UNRELATED: Consultancy: Northwest Biotherapeutics, Comment. Money paid to individual; Grants/Grants Pending: Novocure, Galileo, Comment: Money paid to institution.

\section{REFERENCES}

1. Jain R, Young M, Dogra S, et al. Surprise diagnosis of COVID-19 following neuroimaging evaluation for unrelated reasons during the pandemic in hot spots. AJNR Am J Neuroradiol 2020 May 28. [Epub ahead of print] CrossRef Medline

2. Langenbach MC, Hokamp NG, Persigehl T, et al. MRI appearance of COVID-19 infection. Diagn Interv Radiol 2020 Apr 30. [Epub ahead of print] CrossRef Medline

3. Zubair AS, McAlpine LS, Gardin T, et al. Neuropathogenesis and neurologic manifestations of the coronaviruses in the age of coronavirus disease 2019: a review. JAMA Neurol 2020 May 29. [Epub ahead of print] CrossRef Medline 